\title{
Acceso equitativo al razonamiento científico mediante la tecnología
}

Fair Access to Scientific Reasoning through Technology

Acesso equitativo ao razoamento científico por meio da tecnologia

\section{Leonor Camargo-Uribe* iD o000-0002-2237-7306 Ivonne Twiggy Sandoval-Cáceres** (iD) 0000-0002-7269-8546}

\author{
Artículo de investigación \\ Revista Colombiana de Educación, N.7 73. Segundo semestre de 2017, Bogotá, Colombia. \\ doi: 10.17227/01203916.73rce177.209 \\ Para citar este artículo: Camargo, L., y Sandoval, I. (2017). Acceso equitativo al razonamiento \\ científico mediante la tecnología. Revista Colombiana de Educación, (73), 179-211.
}

\section{(c) (i) (5)}

* Doctora en Didáctica de la Matemática. Profesora del Departamento de Matemáticas Universidad Pedagógica Nacional, Bogotá, Colombia. Correo electrónico: Icamargo@pedagogica.edu.co

** Doctora en Ciencias. Profesora de la Universidad Pedagógica Nacional, México, Ciudad de México. Correo electrónico: isandoval@upn.mx 


\title{
Resumen
}

En la investigación reportada en este artículo se analiza el potencial de un programa de geometría dinámica para promover la aproximación de estudiantes de quinto grado de primaria, de una zona periférica en Ciudad de México, al razonamiento científico. Ellos se enfrentan por primera vez al uso del programa para construir triángulos isósceles y equiláteros, verificar la invariancia de la congruencia de los lados y justificarla usando propiedades de la circunferencia. Con base en elementos de la Teoría de la Variación analizamos las oportunidades que el programa brinda para aprender a pensar y actuar en una sociedad cada vez más influida por los desarrollos tecnológicos. Este es un indicador de equidad. Concluimos que si bien se logran momentos exitosos de acceso al razonamiento científico y los niños pueden proponer vías de construcción y ganan conocimientos que son la base de información compartida, también hay dificultades que erosionan los intentos de lograr equidad tales como la falta de un criterio compartido sobre lo que es una propiedad matemática, la distinción entre cuáles explicaciones son matemáticas y cuáles no y lo que los niños creen que se espera de ellos en la clase de matemáticas

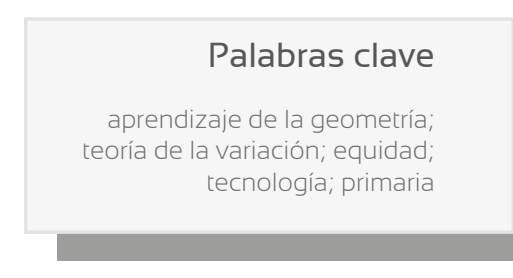

\section{Keywords}

learning geometry; variation theory; equity; technology; elementary school

\begin{abstract}
This paper analyzes the potential of a dynamic geometry software to promote the approach of fifth-grade students from a suburb of Mexico City to scientific reasoning. For the first time, they are faced with using the software to build isosceles and equilateral triangles, to check the invariability of the congruency of the sides, and to justify it using the properties of the circumference. Based on certain elements from Variation Theory, we analyze the opportunities that the software provides to learn to think and act in a society that is increasingly influenced by technological developments. This is an indicator of equity. We conclude that, while successful moments of access to scientific reasoning are achieved and children can propose ways to build and gain the knowledge at the basis of shared information, there are also some difficulties that hinder the attempts to achieve equity, such as the lack of a shared view on what a mathematical property is, the difference between mathematical and non-mathematical explanations, and what children believe
\end{abstract} is expected of them in math class

\section{Resumo}

Na pesquisa reportada neste artigo, analisa-se o potencial de um programa de geometria dinâmica para promover a aproximação de estudantes de quinto ano de educação básica, de uma zona periférica da Cidade do México, ao razoamento cientifico. Eles experimentam por vez primeira o uso do programa para construir triângulos isósceles e equiláteros, verificar a invariância da congruência dos lados e justificá-la utilizando propriedades da circunferência. Com base nos elementos da Teoria da Variação, analisamos as oportunidades que oferece o programa para aprender a pensar e atuar em uma sociedade cada vez mais influenciada pelos desenvolvimentos tecnológicos. Este é um indicador de equidade. Como conclusão, bem se é possível conseguir acesso ao razoamento científico com sucesso por momentos, e as crianças podem propor formas de construção e adquirir conhecimento base da informação compartilhada, também têm dificuldades que estragam os esforços de conseguir equidade, como a falta de um critério compartilhado sobre o que é uma propriedade matemática, a diferença entre quais explicações são matemáticas e quais não, e o que as crianças acham que seja o que se espera deles na aula de matemáticas.

\section{Palavras chave}

aprendizagem da geometria; teoria da variação; equidade; tecnologia; primaria 
Primero pusimos una circunferencia y en esa circunferencia colocamos un radio repitiéndolo dos veces. El triángulo es isósceles porque para donde lo jalemos, siempre dos [lados] son iguales, porque los lados vienen siendo los radios.

Benjamín, $5^{\circ}$ grado

\section{Introducción}

En el marco del proyecto "Geometría: vía al razonamiento científico" (DMA-399-15), equipos de investigación de Colombia y México enfrentamos la tarea de diseñar, implementar y evaluar diversas experiencias matemáticas escolares que promuevan el acercamiento de niños y jóvenes al razonamiento científico y por esta vía impulsen la equidad social. En particular, nos centramos en experiencias matemáticas en las que el acercamiento al razonamiento científico se logre incentivando el uso de programas de geometría dinámica que están hoy al alcance de niños y jóvenes de diversos estratos sociales, por lo menos en las zonas urbanas. Educación gratuita, infraestructura tecnológica, programas informáticos especializados y accesibles y textos escolares gratuitos son elementos necesarios para lograr la equidad social, pero no son suficientes. La equidad también tiene que ver con la calidad de las prácticas escolares, la cual se logra en la medida en que equipos de investigadores y de educadores aunemos esfuerzos para trabajar conjuntamente, en pro de lograr transformaciones de cara a los avances en el campo de la educación matemática.

Hacemos eco de los Ilamados a impulsar la equidad social en el acceso a prácticas poderosas en matemáticas, es decir, a aquellas prácticas útiles para enfrentar retos sociales y culturales situados en el contexto de los sujetos. Vemos esta como una vía para contribuir a cerrar brechas entre la educación proporcionada en países en desarrollo y desarrollados (Forgasz, Vale y Ursini, 2010). Por ello, asumimos la investigación de diseño (Molina, Castro, Molina y Castro, 2011) como una oportunidad para delinear tareas en las que los estudiantes construyan, a partir de la evidencia obtenida por exploración empírica de situaciones de variación, enunciados relativos a fenómenos o hechos abordados en los que argumenten la relación de dependencia de las variables involucradas en ellos.

En este ámbito, el tratamiento de la información y las conclusiones que se obtienen, las cuales muchas veces son logradas con el acompañamiento efectivo del profesor, se constituyen en oportunidades de aproximación al razonamiento científico (Hogan, Nastasi y Pressley, 2000). Aunque no suponemos que se trata del desarrollo del mismo en toda la extensión del término, como resultado del acceso efectivo a un programa de geometría dinámica emergen nuevas prácticas escolares que se apartan de las caracterizadas con la etiqueta "tradicionales". En estas prácticas, los estudiantes tienen la oportunidad de acceder a recursos exploratorios 
para formular conjeturas y a recursos discursivos para argumentar de manera similar a como se explican, defienden y validan afirmaciones en los ámbitos científicos.

En el marco de nuestro objetivo general de investigación, que intenta identificar características de ambientes de aprendizaje para la geometría escolar, mediados por artefactos, que propician el acercamiento de niños y jóvenes al razonamiento científico, en este artículo nos enfocamos en el proceso de resolución de problemas por el que transita un grupo de estudiantes que inicia su último año escolar de primaria, en una zona de la periferia de la Ciudad de México. Identificamos momentos significativos del aprendizaje de los estudiantes mientras resuelven cuatro problemas y analizamos las potencialidades de la tecnología (en este caso de un programa de geometría dinámica) para promover la aproximación al razonamiento científico, mediante el cambio de atención en las representaciones gráficas, y en su manipulación, de la percepción visual hacia el discernimiento. En el estudio nos apoyamos en elementos de la teoría de la variación adaptada por Leung $(2003,2008)$ de la propuesta de Marton, Runesson y Tsui (2004). Este marco nos permite reconocer momentos exitosos que señalan vías de acceso equitativo al conocimiento y también nos llevan a identificar dificultades en el proceso de transitar hacia el razonamiento científico, que erosionan los intentos de lograr la equidad.

\section{Razonamiento científico}

Entendemos el razonamiento científico como el proceso de pensamiento (cognitivo y social) por medio del cual se aborda un fenómeno del campo de las ciencias (o un hecho del campo de las matemáticas) con miras a entenderlo, explicarlo y hacerlo parte del propio bagaje teórico. Está constituido por los distintos procesos de razonamiento que permean las acciones de indagación en el campo de la ciencia, pero que no son exclusivos de ella, pues pueden estar presentes, por ejemplo, en situaciones de la vida cotidiana y profesional. Entre tales procesos figuran la formación de conceptos, la resolución de problemas, el diseño experimental y la puesta a prueba de hipótesis (Dunbar y Klahr, 2013). De dicho proceso destacamos dos acciones: (1) la construcción, a partir de evidencia obtenida por exploración empírica, de un enunciado relativo al fenómeno o hecho abordado, en el que se explicita una relación causal o una relación de dependencia; y (2) la explicación argumentada sobre el asunto que plantea el enunciado. En ambas acciones son transversales distintas maneras de razonar: inducción, abducción, deducción, analogía y correlación. Vemos que una vía para el desarrollo del razonamiento científico puede ser la introducción a una perspectiva teórica de la geometría, máxime porque este es un campo en el que la exploración empírica provee datos que favorecen la elaboración de conjeturas y la justificación. 
Optamos por la conceptualización propuesta en los párrafos anteriores pues tuvimos en cuenta consideraciones del contexto en el que desarroIlamos nuestro ejercicio investigativo. Podría afirmarse que la experiencia matemática previa de los estudiantes participantes había sido muy cercana a la descrita con la etiqueta "matemática tradicional" (modelo de enseñanza expositivo) y, en ese sentido, prácticas como la explicación, la argumentación, la justificación no formaban parte de su actividad habitual en el aula; menos aún concebían una clase de matemáticas en la que pudieran hacer experimentos como parte de la construcción de conocimiento.

\section{Equidad social y acceso equitativo al razonamiento científico con el apoyo de tecnología}

Entendemos la equidad social como igualdad de oportunidades para que niños y jóvenes accedan a formas de actuar en una sociedad cada vez más influida por desarrollos tecnológicos. Ello implica igualdad de acceso a prácticas educativas de calidad, en donde puedan desarrollar estrategias de acción y recursos discursivos para resolver problemas, de manera autónoma, sin importar el estrato social en el cual tienen sus experiencias escolares (Skovsmose, 1985).

La posibilidad de usar programas de geometría dinámica en la enseñanza y el aprendizaje, en tareas cuidadosamente planeadas, ha abierto caminos a la equidad, al generar nuevas y más ricas opciones para acceder al conocimiento matemático y para adentrarse en prácticas de producción de conjeturas y justificaciones, rebasando el universo perceptual en el que usualmente se ubican los estudiantes, cuando se involucran en acercamientos visuales e intuitivos, más alejados del mundo matemático. En las ciencias, y especialmente en matemáticas, la validación está sujeta a la presentación de garantías generales como respaldo a las afirmaciones hechas, que rebasan la experiencia sensorial. La gestión de la tensión entre el funcionamiento en el universo empírico y el teórico de las matemáticas, y en particular de la geometría, es uno de los principales retos de quienes pretendemos impulsar experiencias de aprendizaje significativas, que permitan el desarrollo del razonamiento científico. Se requieren estudios que examinen en profundidad el efecto de esta tensión, sobre la posibilidad real de acceso equitativo de los estudiantes al mundo de la geometría, y por esa vía al razonamiento científico, a partir de situaciones de exploración empírica.

Cuando los estudiantes exploran representaciones geométricas cuya apariencia visual se modifica, mientras que las propiedades características de los objetos representados permanecen invariantes, las representaciones adquieren una nueva dimensión, que va más allá de lo perceptual visual estático y avanza hacia una externalización gráfica de la variación de 
los diferentes atributos que son susceptibles de cambiar, en tanto que las propiedades determinantes permanecen invariables. En ese sentido, la exploración de representaciones en geometría dinámica puede favorecer el descubrimiento y la generalización de propiedades geométricas y de relaciones entre propiedades que surgen de su manifestación en casos particulares, para plasmarse en enunciados generales. Por eso afirmamos, como lo plantean Healy (2000), Jones (2000), Laborde (2000), Mariotti (2000) y Olivero (2003), que los programas de geometría dinámica tienden un puente entre estos dos universos (empírico y teórico) y pueden aprovecharse para favorecer el tránsito entre ellos.

\section{Fases en la resolución de problemas}

Según la teoría de la variación, el aprendizaje de las matemáticas está generalmente relacionado con el proceso de construcción de significado de ideas abstractas que realizan las personas cuando visualizan variaciones de los objetos a las que se refieren, con la esperanza de detectar regularidades y descubrir propiedades matemáticas (Leung y Lopez-Real, 2000). Las propiedades son cualidades invariantes de los objetos o fenómenos, las cuales deben discernirse como parte del proceso de construcción de significado acerca de estos; es decir, deben ser foco de atención por parte de quien construye su significado.

En la teoría de la variación los siguientes supuestos son centrales:

1. El discernimiento de una propiedad se favorece si esta se reconoce como uno de los posibles valores de un atributo; la propiedad se percibe como cualidad común (o igual) a varios objetos de la misma clase en los que varían atributos no relevantes o que no son de interés. Además, se experimentan situaciones de variación en las que el atributo toma otros valores mientras se mantienen invariantes los valores de atributos que no son de interés. Es decir, se construye significado a través de la diferencia.

2. No basta ser informado sobre las propiedades de un objeto para construir significado acerca de las mismas. Por el contrario, el aprendizaje depende de experimentar personalmente la variación de un atributo, diferenciar posibles valores del mismo (o cualidades) e identificar en el objeto el valor específico invariante (o cualidad invariante). Cuando los estudiantes experimentan la variación de esa manera, perciben el atributo como una dimensión de variación.

3. Cuanto mayor sea la diversidad de dimensiones de variación experimentadas en donde se estudien propiedades de un objeto, incluso de manera simultánea, la experiencia de aprendizaje es más rica. Esto es, el significado que se construye del objeto depende de las experiencias de variación que se tengan, en relación con una o más cualidades esenciales de este. 
Para comprender estas ideas, es necesario diferenciar entre atributo, dimensión de variación, cualidad y propiedad. El término atributo se refiere a una característica diferenciadora de los objetos (por ejemplo el color, la temperatura, el área, etc.). La dimensión de variación alude a un rasgo distintivo de los atributos: adoptan más de un valor (son variables); uno de tales valores es la cualidad. Por ejemplo, para el caso del atributo color, cierto objeto tiene la cualidad de ser rojo o de ser amarillo. Una propiedad se refiere a una cualidad del atributo que determina un objeto, por ser una cualidad esencial invariante de este. Una experiencia de variación conduce entonces a discernir una propiedad o más como cualidades esenciales invariantes de una o más dimensiones de variación. Cuando se discierne más de una cualidad se hace referencia a la simultaneidad en la identificación de cualidades esenciales del objeto o fenómeno, lo cual contribuye de manera contundente a la construcción de significado.

Por lo general, en el aula de clase, la construcción de significado de los objetos geométricos se lleva a cabo por medio de la visualización o exploración de representaciones concretas y estáticas que ejemplifican positiva y negativamente los objetos, donde es posible percibir cualidades de estos, como la congruencia de los lados o ángulos, la equidistancia, etc. Pero no se acostumbra involucrar a los estudiantes en actividades matemáticas en las cuales ellos puedan experimentar la variación y reconocer las propiedades de los objetos como cualidades invariantes, como por ejemplo, la longitud, la amplitud angular o la distancia.

Con la introducción de los programas de geometría dinámica en el aula de clase surge una nueva manera de experimentar las dimensiones de variación, el movimiento, que permite establecer propiedades de los objetos geométricos representados, por su invariancia al arrastre de elementos de la representación. Los programas de geometría dinámica proveen una cuasi realidad, de naturaleza euclidiana, corporeizada en la tecnología computacional que explicita dimensiones de variación de atributos, en una experiencia cualitativamente diferente al uso de papel y lápiz, en la que los objetos geométricos se ven en movimiento. Con frecuencia, en lugar de estudiar la congruencia de dos lados en varios ejemplos y no ejemplos de triángulos isósceles, los programas de geometría dinámica permiten la experiencia de arrastrar un vértice de un triángulo isósceles, construido de manera robusta (Healy, 2000), y percibir la cualidad esencial como un invariante al movimiento que produce modificación de las longitudes de los lados. Incluso, la experiencia de arrastrar un vértice de un triángulo construido de manera blanda (Healy, 2000) con la herramienta segmento, procurando que las medidas de las longitudes de dos lados se mantengan iguales, provee una experiencia de control de la cualidad esencial en el curso de la variación, cualitativamente diferente a la experiencia de representar triángulos con dos lados de igual medida, en papel y lápiz. 
Pero aprender a sacar provecho de esta nueva experiencia de variación no es un proceso fácil ni espontáneo. Según Leung (2008), en la resolución de un problema geométrico con el uso de un programa de geometría dinámica los estudiantes enfocan la atención en atributos de las representaciones gráficas y generan estrategias de manipulación sobre estas que avanzan en ciclos de cuatro fases. Leung las denomina: inocencia, adquisición, internalización y trascendencia. Cada fase se diferencia de la otra por el provecho sacado al movimiento por arrastre para experimentar dimensiones de variación, discernir propiedades (aisladas o simultáneamente), y generar con ello una experiencia de construcción de significado. A continuación describimos nuestra interpretación de cada una de ellas.

\section{Inocencia}

Ocurre, o bien cuando los estudiantes se enfrentan a la resolución de un problema usando por primera vez un programa de geometría dinámica; o bien cuando, a pesar de tener alguna experiencia con el programa, es la primera vez que enfrentan la resolución de un problema específico con el apoyo de este. Los estudiantes enfocan la atención en uno o varios atributos de un objeto geométrico, pero quizás vistos de manera independiente y estática, no como dimensiones de variación. En ese sentido, las cualidades no se ven como invariantes al arrastre sino como valores específicos de ejemplos, logrados al hacer una construcción robusta o mediante una construcción blanda que se ajusta mediante el arrastre. Hacer o usar una representación blanda, en la que al arrastrar alguno de sus elementos se pierde la cualidad que se quiere enfocar, puede ser útil para que los estudiantes establezcan un contraste entre aquellas representaciones que tienen la cualidad esencial y aquellas que no la tienen, pero no lo es tanto para separar tal cualidad del objeto y llegar a verla como invariante determinante de este, incluso aunque varíen otros atributos de este con el movimiento.

\section{Adquisición}

Ocurre cuando los estudiantes se familiarizan con algunas de las opciones de construcción del programa de geometría dinámica y en especial con la función de arrastre como herramienta de verificación de la presencia de las cualidades esenciales en una representación, o de generación de una representación que tenga ciertas cualidades impuestas por arrastre. El arrastre de los elementos de una construcción contribuye a contrastar aquellas representaciones en las que la cualidad es invariante al movimiento (y se constituye como propiedad), de las que no. La atención de los estudiantes está puesta en obtener una representación adecuada en el entorno de geometría dinámica y no necesariamente en identificar la cualidad esencial lograda con la representación como un invariante, por lo que no se enfocan en el atributo como una dimensión de variación. 


\section{Internalización}

Ocurre una vez que la familiaridad con las herramientas de construcción del programa de geometría dinámica, especialmente con el arrastre para experimentar mediante el movimiento dimensiones de variación, permite que los estudiantes se enfoquen en la resolución del problema desde el punto de vista matemático y logren la generalización de propiedades del objeto en estudio. Estas son vistas como propiedades invariantes, es decir que no dependen de variaciones de otros atributos, en un continuo de representaciones. Este proceso es el que, desde nuestro punto de vista, da la entrada al mundo teórico, pues los estudiantes comienzan a reconocer las cualidades esenciales como propiedades geométricas generales que determinan los objetos matemáticos. El foco de atención apunta a lo más relevante del problema por resolver, desde el punto de vista matemático.

\section{Trascendencia}

Ocurre cuando los estudiantes se enfocan en la cualidad esencial lograda, independientemente del problema específico resuelto, por lo que el procedimiento se vuelve una herramienta para construir otros objetos geométricos, que comparten la cualidad esencial. Ello permite enfocar la atención en la dimensión de variación de los atributos y en valores específicos de estos en entidades de diversa naturaleza.

De acuerdo con Leung (2008), en el momento de enfrentar un nuevo problema, que implique identificar nuevas cualidades esenciales como invariantes al movimiento por arrastre, los estudiantes transitan por las cuatro fases anteriores, aunque la experiencia acumulada acelera el proceso. Además, en la resolución de un problema, el tránsito de una fase a la otra no es ni simple, ni espontáneo, ni necesariamente secuencial, y los estudiantes pueden incluso regresar a una fase previa, en algún momento, en lugar de avanzar. En este tránsito, la gestión del profesor es central. Él es el encargado de favorecer la atención en el discernimiento de las dimensiones de variación y de las cualidades esenciales, con el objetivo de ir identificando las estrategias útiles para resolver el problema.

\section{Aspectos metodológicos}

Un experimento de enseñanza enmarcada en la investigación de diseño mencionada se llevó a cabo en una escuela pública en la periferia de la Ciudad de México. Se realizaron tres sesiones de clase, cada una de 90 minutos (septiembre 17, 22 y 24 de 2015), al comienzo del año escolar, cedidas por la maestra del curso sexto de primaria, quien es la encargada de impartir todas las clases, excepto Educación Física y Computación. 
El número de estudiantes fluctuó entre 25 y 30 debido a inasistencia de algunos de ellos a algunas sesiones. Los estudiantes trabajaron en grupos de dos o tres, utilizando el programa de geometría dinámica Cabri, instalado en computadores de escritorio.

La meta de la instrucción era que los niños construyeran un triángulo equilátero y justificaran por qué su construcción permitía garantizar que los lados del triángulo eran congruentes. Esperábamos que la congruencia de lados fuera vista por ellos como una propiedad que caracteriza este tipo de triángulos, por lo que la justificación no podía basarse en la percepción visual de la congruencia o en la verificación de la igualdad de medidas en una representación estática, sino en la relación de congruencia de lados invariante ante el cambio en las cualidades de otros atributos de la representación. Por esta razón, decidimos introducir el uso del programa de geometría dinámica Cabri, aunque los estudiantes no lo habían usado previamente. Teníamos como hipótesis de aprendizaje que la producción de construcciones robustas les permitiría no solo experimentar la invariancia de la congruencia de los lados al variar otros aspectos de la representación, sino que las opciones del programa usadas para lograr las construcciones robustas les brindarían las garantías geométricas para lograr justificar la congruencia matemáticamente, y por lo tanto aproximarse al universo teórico de la geometría y, por esa vía, al razonamiento científico. Esta hipótesis nos permitía, además, dar a los niños oportunidades para acercarse a la tecnología y vivir una experiencia que facilitaría un acceso equitativo al conocimiento matemático.

La secuencia de tareas se planeó de tal manera que los dos primeros problemas apuntaran a que los estudiantes identificaran y generalizaran dos invariantes que caracterizan la circunferencia: (1) los puntos de la circunferencia equidistan de su centro, y (2) los segmentos radiales o radios de una circunferencia son congruentes. A medida que iban aprendiendo a usar el programa, esperábamos que consideraran la circunferencia como una opción para construir, verificar y justificar equidistancias entre puntos y congruencias de segmentos. El tercer problema pedía a los estudiantes construir un triángulo isósceles y justificar por qué se podía afirmar que lo era. Y finalmente el cuarto problema pedía construir un triángulo equilátero y justificar la congruencia de los tres lados. La construcción de significado de la congruencia de lados como propiedad que permite caracterizar a los triángulos isósceles y equiláteros se impulsó mediante el aprovechamiento de la función de arrastre para que los estudiantes discernieran y generalizaran la relación geométrica como cualidad invariante.

El plan oficial mexicano de estudios para quinto de primaria (Secretaría de Educación Popular, SEP, 2011, p. 80) señala el siguiente contenido "Distinción entre círculo y circunferencia; su definición y diversas formas de trazo. Identificación de algunos elementos importantes como radio, 
diámetro y centro". Para abordarlo, en los textos oficiales se plantean cinco lecciones. Una de ellas, nombrada "La misma distancia", pretende "Ilustrar a los estudiantes el concepto de circunferencia como el conjunto de puntos que están a la misma distancia de otro punto al que se le llama centro" (SEP, 2013, p. 279). En otra lección se busca que los niños identifiquen que "el círculo tiene un número infinito de diámetros" (p. 284). En diversas tareas se pretende que exploren dichos conceptos y relaciones, usando diversos contextos y problemas. Se pide, por ejemplo, ubicar todos los puntos a la misma distancia de un punto dado, encontrar el centro de una circunferencia usando dos diámetros, construir circunferencias usando el compás e información como la medida del radio o del diámetro. Sin embargo, los conocimientos iniciales sobre la circunferencia que tenían los estudiantes con los que interactuamos no iban más allá del reconocimiento visual de la circunferencia y el radio, como pudimos detectar en los diálogos sostenidos.

Una de las investigadoras actuó como profesora, gestionando las sesiones de clase ${ }^{1}$. La otra investigadora estuvo presente en las tres sesiones de clase, colaboró con el manejo de la cámara de video y en ocasiones interactuó con algunos estudiantes en calidad de otra profesora en la clase. Los enunciados de los cuatro problemas se reportan en el Cuadro 1. Los estudiantes trabajaron en grupos de dos o tres niños, no siempre con los mismos integrantes. Al terminar cada sesión, se hizo una puesta en común, así como al comenzar la segunda sesión, para repasar qué se había aprendido.

Cuadro 1. Enunciados de los problemas propuestos en el experimento de enseñanza

\begin{tabular}{|c|c|c|}
\hline & Problema & Enunciado \\
\hline \multirow[t]{2}{*}{ Pl: } & $\begin{array}{l}\text { Equidistancia } \\
\text { a un punto }\end{array}$ & $\begin{array}{l}\text { Determinar varios puntos que estén a la misma distancia } \\
\text { de un punto dado A. Identificar en qué objeto geométrico } \\
\text { se encuentran todos los puntos que están a la misma } \\
\text { distancia de A. }\end{array}$ \\
\hline & $\begin{array}{l}\text { Congruencia de } \\
\text { radios. Parte } 1\end{array}$ & $\begin{array}{l}\text { Encontrar una propiedad común a todos los radios de una } \\
\text { circunferencia. }\end{array}$ \\
\hline P2: & $\begin{array}{l}\text { Congruencia de } \\
\text { radios. Parte } 2\end{array}$ & $\begin{array}{l}\text { Encontrar qué figura describe el extremo de un segmento } \\
\text { de longitud fija cuando este gira alrededor del otro extremo } \\
\text { que está fijo. Encontrar una propiedad de la figura descrita. }\end{array}$ \\
\hline P3: & $\begin{array}{l}\text { Triángulo } \\
\text { isósceles }\end{array}$ & $\begin{array}{l}\text { Construir un triángulo isósceles, escribir el procedimiento } \\
\text { de construcción y justificar por qué es isósceles. }\end{array}$ \\
\hline P4: & $\begin{array}{l}\text { Triángulo } \\
\text { equilátero }\end{array}$ & $\begin{array}{l}\text { Construir un triángulo equilátero, escribir el procedimiento } \\
\text { de construcción y justificar por qué es equilátero. }\end{array}$ \\
\hline
\end{tabular}

Fuente: elaboración propia.

1 Las investigadoras hicieron las veces de profesoras e interactuaron con los estudiantes con ese rol. La profesora titular observó el proceso, pero no participó en este. En adelante, nos referimos a profesora, pero debe entenderse que se trata de alguna de las profesoras-investigadoras. 
La información empleada en el análisis corresponde a la transcripción completa de las videograbaciones de la interacción comunicativa sostenida entre alguna de las profesoras y los grupos de estudiantes, así como en la puesta en común. Por lo general, al dirigirse a un grupo, la profesora comenzaba el diálogo pidiendo a los niños que narraran lo que habían hecho e incluso les pedía que hicieran una reconstrucción de las acciones realizadas con el programa de geometría dinámica. También se recogieron las hojas en las que algunos estudiantes reportaron la resolución de los problemas P3 y $\mathrm{P} 4$.

Los datos para el análisis corresponden a fragmentos representativos de interacción comunicativa, en los que es posible reconstruir las estrategias seguidas por los niños y la mediación de la profesora. Empleamos las fases sugeridas por Leung (2000) para organizar la descripción y el análisis de la resolución de cada problema, visto cada uno como un ciclo de aprendizaje. Estas fases nos permiten señalar aspectos críticos del aprendizaje de la geometría y del avance en el razonamiento, con el apoyo del programa de geometría dinámica. En busca de indicadores de equidad, rastreamos qué estrategias de acción se constituyeron para los estudiantes en recursos de exploración y construcción activa de conocimiento, qué experiencias les permitieron avanzar hacia el discernimiento de propiedades geométricas, qué acceso tuvieron a recursos discursivos para construir argumentos propios, comunicar su razonamiento y confrontarlo con los de sus compañeros y qué muestras dan de trabajo autónomo para resolver los conflictos que se les presentan.

Optamos por emplear la técnica de viñetas para presentar el análisis. En ese sentido, hacemos una reconstrucción de lo sucedido en cada sesión, articulando descripciones con interpretaciones e inferencias del aprendizaje de los estudiantes, a partir de la teoría. Dondequiera que vemos oportuno hacerlo, incluimos fragmentos de interacción a manera de diálogo, o únicamente reconstruimos los hechos.

\section{Análisis}

En las producciones de los estudiantes identificamos, en cada fase, las maneras como ellos experimentan la variación y construyen el significado de las propiedades de equidistancia y congruencia al resolver los problemas. A continuación exponemos el análisis realizado, centrándonos en el tránsito de los estudiantes por las diferentes fases de la resolución de los problemas P1 y P3 -no necesariamente todas, pues no siempre recorrieron las cuatro fases. 


\section{Problema 1: Equidistancia a un punto dado $A$}

Se propone el problema a los estudiantes después de un corto tiempo en el que, por solicitud de la profesora, ellos despliegan las ventanas del programa Cabri para leer las opciones que hay en cada una de ellas; luego buscan cómo determinar diez puntos en la pantalla y los nombran con letras mayúsculas de la $A$ a la J. Mientras que los estudiantes trabajan en parejas o tríos, la profesora interactúa con ellos para darles indicaciones, en caso necesario, hasta que todos los grupos logran determinar diez puntos, nombrarlos y arrastrarlos por la pantalla usando la herramienta apuntador. Después, la profesora les indica la tarea: "Colocar todos los puntos de manera que queden a la misma distancia del punto $A^{\prime \prime}$.

\section{Fase de inocencia}

Casi todos los grupos de estudiantes interpretan la instrucción "a la misma

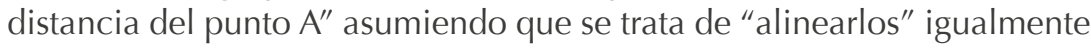
separados, comenzando por $A$ y terminando en $I$. Por eso ubican los puntos en fila, entre $A$ y $J$, tomando como referencia la posición de $A$ y $B$ de para localizar los demás puntos alineados a estos, igualmente separados unos de otros y en orden alfabético. Algunos grupos realizan una configuración inicial un poco diferente, al proponer dos "filas" de puntos igualmente separados entre sí en cada fila, de tal suerte que los puntos $A, F, H, I, I$, están más o menos en la misma línea e igualmente separados, los puntos $B, C, D, E, G$, parecen estar en otra línea y las distancias entre dos puntos de una fila parecen reproducirse en los puntos inmediatamente debajo (Figuras 1a y $1 b$ ).

Probablemente las experiencias previas de los estudiantes relacionadas con la igualdad de distancias, tanto al hacer filas en el patio como en tareas que implican el uso de una regla graduada, tienen que ver con establecer la misma distancia entre cada dos puntos consecutivos de la secuencia y no a la misma distancia a uno dado.

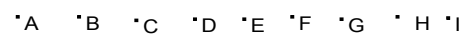

Figura 1a

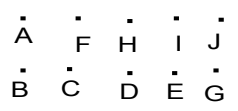

Figura $1 b$

Por ser la primera vez que usan el programa de geometría dinámica, lo emplean como si estuvieran trabajando en papel y lápiz, determinando los puntos donde ellos consideran que se cumple la relación pedida. Aunque mueven los puntos, esta acción está relacionada más con dibujar 
y borrar, para lograr la equiseparación, que con la intención de hacer variar la dirección, la colinealidad, la interestancia o la distancia que los separa. No se sorprenden por poder arrastrarlos. Su atención está puesta principalmente en lograr una cierta ubicación; no se enfocan en la función de arrastre, y lo que ellos consideran como equidistancia se ilustra con los puntos ubicados en posiciones como las que muestran las figuras $1 \mathrm{a}$ y $1 \mathrm{~b}$.

La profesora interactúa con los estudiantes, con el propósito de lograr que empiecen a ver el arrastre como recurso de exploración y centren la atención en el punto $A$, que debe servir de referencia para la ubicación de los demás puntos, pues todos deben quedar equidistantes de $A$. A unos estudiantes les pide que muevan a $A$ hacia un lugar céntrico de la pantalla, distinto del que estaba, y luego que muevan los demás puntos "hasta que queden a la misma distancia del punto $A^{\prime \prime}$. A otros les pide que señalen con el dedo la distancia del punto $A$ al punto $B$ y la distancia del punto $A$ al punto $D$; luego les indica que arrastren los puntos hasta lograr que ambas distancias sean iguales. Desde el punto de vista de la teoría de variación, ella pretende generar oportunidades para impulsar un avance hacia el discernimiento de la relación de equidistancia a un punto, evocando las experiencias cotidianas y escolares de los niños sobre distancias entre puntos (trayectorias recorridas, trazos de caminos, ubicación en un plano cartesiano) para que interpreten la relación "misma distancia a un punto" como diferente de "misma distancia entre puntos".

Las indicaciones de la profesora tienen efectos diferentes en los niños y conducen a estrategias de acción distintas. Por ejemplo, Samanta (quien había hecho la configuración inicial de la figura 1a) arrastra el punto $A$ y lo ubica hacia el centro de la pantalla. A continuación, arrastra los puntos $B, C, D \ldots$ hasta que logra una configuración muy parecida a la anterior, determinada por la nueva posición del punto $A$ y por un mayor cuidado respecto a la colinealidad y a la distancia entre dos puntos "consecutivos" en la secuencia de puntos. Su compañero, Gustavo, señala los puntos $A$, $B$ y $E$ y dice: "tenemos que tener todos los puntos a la misma distancia de $A$, pero ahí no es". La profesora opta por pedir a la niña que ubique a $B$ en una posición no alineada con los demás y luego les pide que "ubiquen a $C$ a la misma distancia de $A$ que como está $B^{\prime \prime}$. Con ello busca que la pareja de puntos $A, B$ se destaque, para que Samanta se enfoque en la distancia entre ellos y la use de referencia para ubicar los otros puntos a la misma distancia. Esta intervención intenta impulsar el discernimiento de la relación de equidistancia, con el apoyo del cambio de posición de puntos. Samanta parece haber captado la idea pues ubica a $C$ perceptualmente a una distancia de $A$ similar a la distancia $A B$ como se muestra en la figura 2. Ella comienza a comparar perceptualmente la distancia de $A$ a $B$ con la de a otros puntos y no cada dos puntos entre sí. 


\section{$\dot{A} \quad \dot{C} \quad \dot{D} \quad \dot{E} \quad \dot{F} \dot{G} \quad \dot{H}$ \\ $\dot{B}$}

Figura 2

Javier, quien parte de la figura $1 \mathrm{~b}$, ubica a $C$ perceptualmente a la misma distancia de $A$ como está $B$. Parece que logra interpretar de manera apropiada la relación de equidistancia, cambiando la posición de $C$ a un lugar en el que la relación de equidistancia se verifica. Pero cuando la profesora le pide ubicar otro punto que esté a la misma distancia, lo ubica superpuesto a $C$. Quizás prima la idea de alineación y no se le ocurre que los puntos que equidistan de $A$ pueden no estar alineados. En términos del uso del programa, el estudiante no sabe aún que si dos puntos se superponen, representan el mismo punto, geométricamente hablando. Por solicitud de la profesora, busca posiciones que "estén a la misma distancia, pero no encima". Arrastra los puntos $E$ y $D$, y propone una configuración como la de la figura 3 b. Los puntos $B, C, D$ y E están aproximadamente a la misma distancia de $A$ mientras que los demás no. La configuración ilustra el contraste entre "misma distancia" y "diferente distancia". Javier termina de arrastrar los puntos y logra una configuración como la de la figura 3c. Poco a poco, mediante una construcción activa, los grupos van obteniendo configuraciones similares.

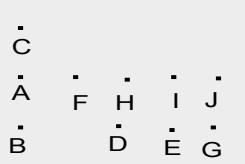

Figura 3a

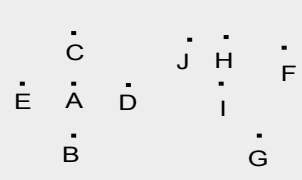

Figura 3b

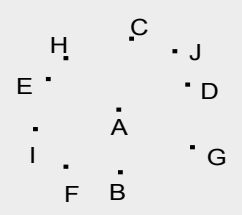

Figura 3c

\section{Fase de adquisición}

Cuando los estudiantes entrevén que los puntos tienen una disposición circular, la profesora los invita a buscar una herramienta del programa que les permita construir circunferencias. Algunos estudiantes juegan con la opción "círculo", para instrumentalizar su funcionamiento y volverla una herramienta de exploración. Apoyados por las instrucciones de la profesora, o de compañeros a quienes ella les ha dado indicaciones, construyen una circunferencia con centro en $A$ y radio $\overline{A B}$. Luego, por sugerencia de 
la profesora, "acomodan" los demás puntos para que la representación quede "mejor". Ellos colocan los puntos para que perceptualmente se vean como si estuvieran en la circunferencia (figura 4).

La acción que detona el cambio de fase es la ubicación de algunos puntos alrededor del punto $A$ y no alineados, lo que hace más evidente el contraste entre aquellos puntos que están a la misma distancia de $A$, de los que no lo están. Esto les da una idea a los estudiantes sobre cómo resolver el problema aprovechando la opción que brinda el programa Cabri. La atención se enfoca en lograr la representación adecuada. Se dan cuenta de que la circunferencia les permite ubicar varios puntos equidistantes de $A$ como $B$, pero aún no usan el arrastre para explorar o verificar relaciones geométricas y no identifican la equidistancia como una relación que hay que preservar, pese a que otros aspectos de la representación cambien. En ese sentido, los estudiantes aún no ven la circunferencia como un lugar geométrico, no generalizan la propiedad de equidistancia a un punto como propiedad de cualquier punto de la circunferencia $y$, adicionalmente, quedan satisfechos con el ajuste perceptual. Aún no diferencian entre una construcción blanda y una robusta. La profesora interviene para centrar la atención en la inestabilidad de la construcción realizada y por lo tanto en las variaciones que pueden experimentar las distancias entre los puntos. Les pide arrastrar el punto $A$. Algunos estudiantes, como Javier, arrastran levemente el punto $A$, anticipando que la mayoría de puntos quedarán fuera de la circunferencia. Su compañera Estefanía, lo tranquiliza: "Ahorita los movemos", dándole a entender que podrán recuperar la configuración arrastrando los puntos. Ella da muestras de autonomía para resolver el problema que enfrentan.

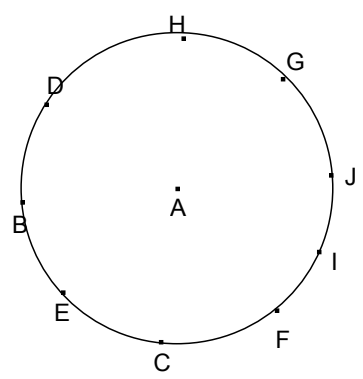

Figura 4

Aunque la atención de los estudiantes parece estar puesta en la ubicación de puntos sobre la circunferencia construida, su acción se constituye en una experiencia exploratoria en la que relacionan la posición de y con la posición que deben tener los demás puntos que están a la misma 
distancia de que. Es evidente que los estudiantes contrastan cuándo se tiene y cuándo no se tiene la equidistancia, pero no saben aún cómo lograrla como una propiedad. Probablemente tienen un significado de la situación relacionado con la desorganización perceptual de la representación con la que estaban haciendo ostensiva la equidistancia, pero no con el hecho de no haberse apoyado en propiedades geométricas de objetos para hacer la construcción. Esta es una estrategia de acción que debe aprenderse al usar un programa de geometría dinámica, pues solo las construcciones robustas mantienen invariantes las cualidades esenciales cuando los elementos de la construcción se arrastran.

La profesora explica a todo el curso cómo construir un punto nuevo que "pertenece" a la circunferencia; enfatiza en que el punto no debe quedar adentro sino en la circunferencia y les pide fijarse en el letrero que sale en la pantalla de Cabri al acercar el apuntador a aquella. Luego, les pide que contrasten el comportamiento del punto, construido en la circunferencia, con el de otros puntos, cuando los arrastran. El movimiento de $P$ acapara la atención de los estudiantes, quienes difícilmente perciben la "misma distancia" de cada punto de la circunferencia al punto . no es visto como cualquier punto de la circunferencia (una variable), sino como un punto que se mueve en una trayectoria específica.

En busca de reorientar la atención en la equidistancia, la profesora pregunta por la característica del movimiento de. Como ningún estudiante responde, ella dice: "[] se mueve y siempre está a la misma distancia de ". Les pide borrar toda la construcción, comenzar por una circunferencia y determinar varios puntos que pertenezcan a ella. Una vez construidos varios puntos en la circunferencia, ella insiste en enfocarse en la propiedad de interés: equidistancia. Los estudiantes consideran que los puntos construidos tienen dos propiedades: están en la circunferencia y se mueven en ella. Solo por insinuación de la profesora identifican que están a la misma distancia del punto . La experiencia del arrastre desplaza la atención de la distancia al movimiento. Quizás si la profesora les hubiera sugerido mover algunos puntos, buscando una relación entre las distintas posiciones de estos y el punto, los estudiantes podrían haber recuperado el foco de atención en la equidistancia de los puntos a . Pero la pregunta por una "propiedad" o "característica" no logra cambiar el foco de atención. Para que se fijen en tal relación les pregunta directamente por la equidistancia con respecto a cuál punto. Javier da la respuesta que ella espera: "del punto $A^{\prime \prime}$. En términos de la resolución del problema, los estudiantes cumplen la misión encomendada pues representan puntos que equidistan de, en una construcción robusta. Además, usan el arrastre como herramienta de verificación de una propiedad invariante, como es la trayectoria circular de los puntos. Pero no han fijado la atención en la equidistancia como invariante característico. 


\section{Fases de internalización y trascendencia}

La actividad matemática de los estudiantes relacionada con el problema de identificar el lugar geométrico de puntos que equidistan de un punto dado llegó en ese momento hasta la fase de adquisición. No hubo lugar a experiencias de variación que permitieran separar la equidistancia como un valor específico de la relación entre los puntos de una circunferencia y el centro de esta ni a generalizar la equidistancia como un invariante de la circunferencia. En una puesta en común, que sucede después de trabajar en el segundo problema, la profesora intenta suplir esta deficiencia mediante un ejercicio de ubicación de los estudiantes en el salón. Propone una tarea similar a la sugerida en las lecciones del texto guía, aunque previamente este no se había revisado. Pide a los estudiantes ubicarse a la misma distancia de un niño seleccionado. Los niños se ubican alrededor, formando una circunferencia y la profesora les pregunta por una característica común a todos los puntos de una circunferencia. Dónovan responde: "están a la misma distancia". Sin embargo, creemos que los estudiantes siguen enfocados en la manifestación perceptual de la equidistancia, porque aún no se ha introducido el uso de la medida. A pesar de ello, la experiencia desarrollada brinda a los niños oportunidades para discernir mediante un proceso de exploración con herramientas útiles (como el arrastre y la construcción de circunferencias), comunicar sus hallazgos y resolver con autonomía situaciones problemáticas. Estos son algunos de los indicadores de equidad encontrados en esta parte de la experiencia.

\section{Problema 3: construcción de un triángulo isósceles}

Dado el interés por dar a los estudiantes oportunidades de explorar, discernir y argumentar, el énfasis del problema P3 está en el proceso de construcción de un triángulo isósceles y en la justificación de por qué es isósceles, y no en llegar a la definición de este tipo de triángulo. Por ello, antes de comenzar a resolverlo, la profesora con intervenciones de algunos estudiantes, reconstruye las definiciones de triángulo equilátero, isósceles y escaleno.

\section{Fase de inocencia}

Si bien los estudiantes han ganado cierta experiencia en el uso del programa de geometría dinámica, en este nuevo problema transitan otra vez por esta fase. No obstante, el arrastre cambia de funcionalidad. Se usa para obtener un ejemplo, mediante una representación estática, que perceptualmente cumple la propiedad de congruencia de dos lados. 
La herramienta de arrastre se complementa con la de medida, un nuevo recurso de exploración, para lograr una posición en la que dos de las medidas coincidan o para verificar tales medidas.

Todos los grupos hacen construcciones blandas, a partir de tres segmentos concatenados, cuyas longitudes muestran en la pantalla o a partir de la opción "Triángulo"; usan el arrastre para lograr que dos lados midan lo mismo. Esta experiencia permite a los estudiantes contrastar representaciones que tienen la propiedad de congruencia de dos lados de las que no, experimentando la congruencia como dimensión de variación. Incluso, la atención puesta en lograr dos lados de igual longitud les hace perder el interés por la posición espacial, ampliando el universo de ejemplos con representaciones en posiciones no estándar. Sin embargo, los estudiantes no buscan un procedimiento que les permita hacer una construcción robusta, por lo que aún no identifican la congruencia como cualidad esencial invariante de un triángulo isósceles. En la figura 5 se muestran dos ejemplos de representaciones.
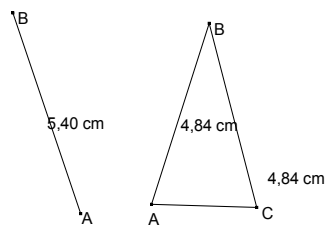

Equipo de Carolina y Samanta

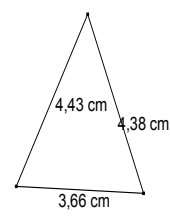

Equipo de Teresa y Belén

Figura 5

El uso simultáneo del arrastre y la medición les da retroalimentación inmediata y útil a algunos grupos y les permite hacer los ajustes necesarios y lograr una representación de un triángulo isósceles. Algunos ejemplos de sus descripciones son:

Teresa: Primero pusimos tres segmentos [...] y ya después [...], los acomodamos de manera que dos quedaran iguales y uno quedara diferente.

Ariel: Estamos tratando de hacer un triángulo isósceles. Para saber si [dos lados] miden lo mismo, le ponemos aquí [señala la opción "Distancia y longitud"] para medir los [lados] azules [del triángulo]; [toma la medida de los tres lados]. Y si no miden igual, los vamos acomodando. [Arrastra un vértice hasta lograr dos lados de igual longitud.]

Otros grupos tienen dificultades con la instrumentalización de la opción "Distancia y longitud". Este es el caso de Guadalupe y Joshua, que no usan adecuadamente la opción, pues han construido el triángulo 
usando la opción "Triángulo del menú" y entonces el programa provee el perímetro. Sin embargo, ellos afirman que dos lados son iguales, sin apoyarse en la medida como "garantía" de esta afirmación. La profesora promueve una conversación al respecto.

Profesora: [En la pantalla tienen dibujado un triángulo con apariencia de isósceles, pero solo aparece la medida del perímetro.] ¿Ese triángulo es isósceles, Guadalupe?

Guadalupe: Sí.

Profesora: ¿Cómo sabes?

Guadalupe: Porque tiene sus dos partes iguales.

Profesora: $\quad$ ¿Y cómo sabes que tiene sus dos partes iguales? [...] ¿Las midieron? [...] Yo solo veo una medida [...] están midiendo el perímetro. Acércate [con el mouse] y mira lo que dice ahí, cuando sale el letrerito rojo: "perímetro". Ese no es el lado. [La profesora les enseña cómo medir un lado] Este ladito mide 7,24 [] y este [] 7,23. Parece isósceles [...].

Para Guadalupe, el hecho de no obtener sino un número al activar la opción "Distancia y longitud" no constituye un problema, ni se interesa por averiguar qué información brinda ese número. Quizás usó la opción por insinuación de la profesora o de algún compañero. Pero ella basa la identificación de la congruencia de los lados simplemente en la apreciación perceptual de la igualdad de medidas.

La profesora objeta esta manera de obtener información y le pregunta si ella midió los lados. Pero como de inmediato se da cuenta de que los estudiantes han empleado erróneamente la opción que brinda el programa, ella se centra en explicar el funcionamiento de la herramienta "Distancia y longitud" y no en discutir con ellos qué información se obtuvo. Cabe señalar que cuando se trabaja con lápiz y papel el perímetro de un polígono no se obtiene haciendo una única medida, sino determinando las medidas de los lados y luego sumándolas. En ese sentido, el resultado numérico obtenido por Guadalupe y Joshua es quizás considerado como la medida de un lado. Pero como no pueden obtener la otra medida, desisten del intento y toman la decisión con base en la percepción.

Otro tipo de intervenciones de la profesora tienen el propósito de introducir el arrastre como herramienta de verificación de las propiedades que se dice que tiene la representación hecha. Es su manera de guiar el avance hacia el análisis de las cualidades esenciales del triángulo isósceles como invariantes al arrastre. Estas intervenciones suceden cuando los 
estudiantes, a partir de que ella lo sugiere, arrastran vértices del triángulo construido y se pierde la congruencia de los lados. Estas son algunas de las intervenciones dirigidas hacia algún estudiante:

Ejemplo 1. [Profesora a Guadalupe]: ¡Ahí ya no [es isósceles]! Entonces necesito construir uno que por más que lo movamos para todos los lados, [estos] sigan iguales [en su medida] ¿Qué se les ocurre hacer?

Ejemplo 2. [Profesora a Ariel]: [...] no está bien diseñado porque si mueves un vértice se te daña. Necesito buscar otro camino para hacerlo sin que se dañe nunca, nunca, nunca ¿Saben cómo? [...] [...] A algunos niños se les ocurrió usar una circunferencia. Miren a ver si usando una circunferencia, pueden construir un triángulo que siempre sea isósceles. ¿Sí?

Ejemplo 3. [Profesora a Uriel]: Eso que tú trataste, que te queden dos lados iguales, se daña. Entonces tenemos que inventarnos otra construcción de tal modo que cuando yo pase, y les mueva, siempre va a tener dos lados iguales ¿Qué se les ocurre que podemos hacer? [...] A Belén se le ocurrió hacer una circunferencia. Vamos a ver. Haz una circunferencia Uriel.

Según la teoría de variación, los diferentes grupos de estudiantes están discerniendo la cualidad esencial del triángulo isósceles: dos lados congruentes. Han experimentado el contraste entre representaciones que tienen la cualidad y las que no. Pero no han generalizado la propiedad y siguen interesados en casos específicos.

La profesora les sugiere usar una circunferencia para ver si logran hacer una construcción robusta. Ubicamos las primeras estrategias en las que intentan usarla en la fase de inocencia, pues los estudiantes no se han valido de esta opción previamente y no saben cómo hacerlo. La profesora supone que establecerán rápidamente la asociación entre la congruencia de los radios de una circunferencia y la congruencia de los lados del triángulo isósceles y se valdrán de ello en su construcción. Sin embargo, no todos los grupos establecen tal asociación. En la figura 6 presentamos algunas propuestas.

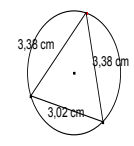

Teresa y Belén

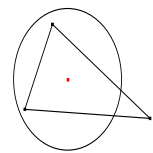

Uriel y Sandra

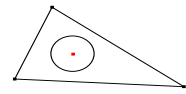

Ariel y Carolina

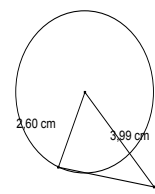

Andrés y Aldo 
Teresa y Belén, por ejemplo, explican a la profesora lo que quieren hacer:

Teresa: Vamos poniendo tres puntos de forma que queden en un triángulo, sobre la circunferencia para que ya dé $[\ldots]$ y no se dañe.

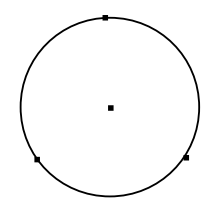

Teresa: [Hace un triángulo como el de la figura, usando los puntos que había determinado en la circunferencia y la opción "segmento"].

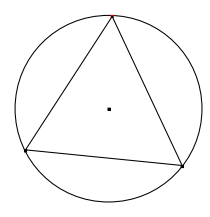

Profesora: Un momento, $[\ldots]$ ¿Ustedes creen que ese triángulo es isósceles?

Belén: ¡No!

Profesora : ¿No? ¿Entonces por qué lo construyeron?

Teresa: $\quad$ Porque ya después lo vamos a medir.

Las estudiantes continúan tratando de lograr un ejemplo representativo, pero no general. No emplean las propiedades de los radios de la circunferencia para lograr la construcción robusta y pretenden basarse en las medidas de los lados para lograr la congruencia de los lados.

Una construcción en la que aparentemente se usan dos radios de la circunferencia como lados del triángulo es la de Andrés y Aldo. Sin embargo, en la explicación que hace Aldo menciona que determinó la medida de dos lados, y luego arrastró uno de los vértices hasta lograr la igualdad. Después construyó una circunferencia con centro en un vértice y usó un lado del triángulo como radio. Al hacer el arrastre del tercer vértice, se hace evidente que este no se construyó en la circunferencia sino que la ubicación se ajustó mediante el arrastre. La profesora les sugiere que comiencen de nuevo, haciendo una circunferencia como primera construcción. 
En las descripciones que hacen los diferentes grupos, cuando la profesora les pide explicar lo que han hecho, se evidencia contraste entre aquellas representaciones de un triángulo isósceles de las que no lo son. Logran proponer configuraciones empleando las opciones de medida y arrastre, pero ninguno propone una construcción robusta. Como no han separado la congruencia como cualidad de diversos segmentos, no asocian la congruencia de radios de una circunferencia con la congruencia de lados de un triángulo. Tampoco han generalizado la propiedad, independizando la congruencia de las medidas específicas obtenidas en casos particulares.

\section{Fase de adquisición}

Los estudiantes requieren de la mediación de la profesora para avanzar hacia una construcción robusta que les permita percibir la propiedad esencial de los triángulos isósceles como un invariante al arrastre. Para ello, deben poner en juego el hecho geométrico visto en el problema anterior. Además de pedir a los estudiantes que arrastren los vértices de los triángulos construidos para ver que la construcción no conserva la cualidad esencial al arrastre, los invita a pensar en cómo proceder: "Tenemos que pensar en una forma que nunca se nos dañe el triángulo isósceles. ¿Qué se les ocurre que podemos hacer?" “Para qué nos serviría una circunferencia?" Con ello promueve que los niños busquen por sí mismos la solución al problema.

Sandra y Aldo, aunque tímidamente, sugieren cómo proceder. Sandra le dice a Benjamín, su compañero: "Que dos de sus lados sean radios", pero Benjamín construye un triángulo inscrito en una circunferencia sin considerar la idea propuesta. Sandra ha encontrado la manera de proponer una construcción robusta que le permite resolver el problema. Le falta hacerse escuchar y convencer a su compañero. Por su parte, Aldo, atendiendo a la sugerencia dada por la profesora de comenzar por una circunferencia, la construye y luego propone: “Unir los puntos y unir los [...] [señala con los dedos índice y pulgar el centro de la circunferencia y un punto en la circunferencia]". Hace algunos intentos de determinar puntos en la circunferencia, luego desiste de esa idea, activa la opción "Segmento" y construye una cuerda de la circunferencia. Finalmente, traza los radios correspondientes a los extremos de la cuerda.

Después de varios intentos y a partir de observar y confrontar los resultados obtenidos unos y otros, varios grupos usan la circunferencia y dos de sus radios para construir un triángulo isósceles. La atención está puesta en lograr hacer la construcción robusta. Algunos se valen del arrastre para verificar, pero espontáneamente no justifican la congruencia de los lados del triángulo. 
-En la figura 7 se muestran dos ejemplos de las construcciones que hicieron, en dos momentos del proceso.

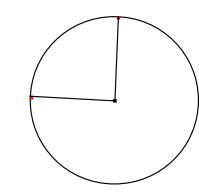

Carolina y Guadalupe; Estefanía, Eric y Graciela; Teresa, Uriel y Belén

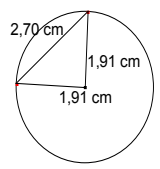

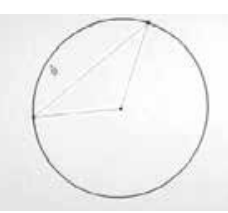

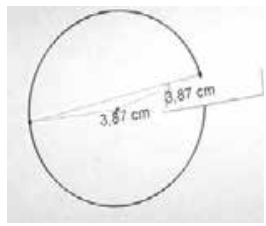

Sandra y Benjamín; Joshua y Esmeralda

Figura 7

A pesar de haber hecho la construcción robusta que resuelve el problema y haber verificado por arrastre que los lados son congruentes, Carolina propone tomar medidas. La profesora la interroga sobre la necesidad de hacerlo.

Carolina: Aquí hay una forma [señala los menús] para saber cuánto miden.

Profesora: ¿ ¿Necesitamos saber cuánto miden?

Carolina: Para saber si es la misma medida del otro. (Guadalupe exhibe la medida de los radios.)

Profesora: ¿ ¿Y no sabíamos ya que tienen la misma medida? ¿Sí o no? Mueve los vértices [...] Mueve más [...] Mueve más. ¿Qué pasa con los lados?

Carolina: Se hacen más grandes.

Carolina percibe el cambio en las longitudes de los lados, pero no se centra en la invariancia de la congruencia. No ha separado esta propiedad como cualidad esencial por atender. Por eso considera que es necesario medir para verificar que el triángulo es isósceles.

Sandra logra que Benjamín haga la construcción del triángulo, a partir de una circunferencia y dos radios. Ella ha anticipado que así logrará hacer la construcción: "Este va a ser siempre un triángulo (señala los dos radios y con el dedo recorre el trayecto del tercer lado)". La profesora le pregunta por el tipo de triángulo y ella exclama: ¡Un isósceles! Sandra ha logrado formular una conjetura cierta, resultado de un proceso de exploración y descubrimiento. Pero su compañero, Benjamín, no está de acuerdo. Dice que el triángulo construido no siempre es isósceles y hace un arrastre 
cuidadoso de los vértices, quizás en busca de una configuración que le sirva como no ejemplo. Luego obtiene las medidas de los lados y arrastra los vértices del triángulo para verificar que son iguales.

Aunque algunos grupos han logrado resolver el problema, no podemos afirmar que han pasado a la fase de internalización. Siguen viendo la situación desde un punto de vista empírico y no dan muestras de generalizar. Por eso sus explicaciones están basadas en describir lo que hicieron para construir el triángulo. La siguiente conversación es un ejemplo de ello.

Profesora: ¿YY por qué [los lados] son de la misma longitud?

Graciela: Porque son del mismo tamaño.

Eric: $\quad$ Porque $[\ldots]$ este $[\ldots]$ los pusimos a la misma distancia. [...] [...] Porque los medimos.

Profesora: ¿Los midieron para ponerlos a la misma distancia? No, eso no fue lo que me contaron. ¿Cómo fue que hicieron la construcción? [...] [...] ¿Qué fue lo primero que hicieron? Vuelve a hacer la construcción. [...] [...] ¿Qué fue lo primero que hicieron?

Eric: Hicimos un círculo. [Construye una circunferencia].

Profesora: ¿Y para qué hicieron el círculo?

Eric: $\quad$ Para poder guiarnos.

Profesora: ¿Guiarnos de qué?

Eric: $\quad$ Para hacer las líneas de la distancia. [Construye un radio de la circunferencia].

Profesora: ¡AAh! Es que la utilizaron para saber que las líneas, los segmentos, medían lo mismo. ¿Y por qué saben que los segmentos medían lo mismo? [...] [...].

Eric: $\quad$ Porque para [...] [Construye el lado del triángulo que es cuerda de la circunferencia].

Profesora: ¿Qué fue lo que dijimos de las propiedades de la circunferencia? [...] ¿Ah? [...] ¿Eric?

Eric: [Construye el tercer lado del triángulo, haciendo otro radio.] Que todos los puntos están a la misma distancia de [...]. 
Graciela y Eric reconstruyen el proceso desarrollado para obtener el triángulo isósceles. Al explicar el porqué de la congruencia de los lados, Graciela alude a que tienen el mismo tamaño y Eric a la equidistancia y a la igualdad de medida. Las explicaciones dan a entender que hicieron un triángulo y luego ajustaron las longitudes de los lados para que quedaran iguales, aunque el proceso que llevaron a cabo no fue así. Ellos no establecen la diferencia en el resultado que se obtiene si primero hacen el triángulo y luego una circunferencia para ajustar las medidas de dos lados, o si primero hacen una circunferencia y con base en los radios de esta obtienen los lados del triángulo. La profesora intenta que capten la diferencia pidiéndoles reconstruir el triángulo. También busca que los estudiantes pasen de un argumento empírico a un argumento teórico, para justificar la congruencia, de tal suerte que avancen hacia la generalización. Pero con esta conversación no lo logra.

\section{Fase de internalización}

La profesora busca que los estudiantes diferencien la congruencia de los lados de otras cualidades de las representaciones, generalizándola como propiedad invariante de cualquier triángulo isósceles. Con intervenciones como las siguientes, busca que los estudiantes avancen hacia la internalización.

Ejemplo 1. [Profesora a todo el grupo]: Tenemos que hacer un triángulo que sea isósceles y que, no importa para donde lo muevan, se tiene que quedar con una propiedad. ¿Con cuál propiedad se tiene que quedar?

Ejemplo 2. [Profesora a Teresa y Uriel]: [...] Tienen que explicarme por qué ese lado [señala un radio] y ese lado [señala el otro radio], siempre van a ser iguales.

Ejemplo 4. [Profesora a Joshua y Esmeralda] Claro, porque hicimos radios de la circunferencia que siempre son iguales.

En dos parejas se aprecia un avance de algunos estudiantes hacia la generalización de la propiedad. Una de ellas es la de Teresa y Belén. Así explican a la profesora:

Teresa: $\quad$ Y ya de cualquier manera que lo pongamos siempre son iguales.

Profesora: A ver, muéstrenme. Agranda y achica el círculo, arrastra los vértices.

Teresa: $\quad$ Porque los dos están a la misma distancia del centro. 
Profesora: ¿Y por qué sabes eso?

[...] Belén: Porque siempre va a ser [...] todos los [señala un radio con el dedo].

Profesora: radios.

Belén: $\quad$ Son del mismo tamaño.

Las dos intervenciones de Teresa no se refieren a un caso particular. Ella generaliza la situación aludiendo a que los lados "siempre son iguales", alude a que dos vértices del triángulo "están a la misma distancia del centro" e intenta hacer referencia a la propiedad común de todos los radios de una circunferencia. Este es un ejemplo de construcción activa de conocimiento, donde la exploración e intercambio en el grupo permitió avanzar hacia la diferenciación de propiedades geométricas. Belén la apoya en la enunciación del hecho general, pues Teresa olvida cómo se denominan los radios. La experiencia de resolución del problema les ha permitido discernir la propiedad esencial de los triángulos isósceles como invariante en una construcción dinámica. Además, ha llevado a Teresa a justificar teóricamente la congruencia, aproximándose al mundo geométrico.

Esmeralda y Joshua parecen separar la congruencia como propiedad invariante ante el movimiento. Sin embargo es difícil identificar hasta qué punto han entendido la propiedad, pues usan un lenguaje poco claro. Cuando la profesora le pregunta a Esmeralda qué pasa si se agrandan o achican la circunferencia Esmeralda dice que los radios "se hicieran más grandes pero seguirían en el mismo porcentaje", probablemente aludiendo a la congruencia. Joshua la apoya: "Si lo mueves y siguen los mismos centímetros, es que sí es isósceles".

El siguiente diálogo entre la profesora y Carolina da muestra de un arribo de la estudiante a la fase de internalización.

Profesora: ¿YY por qué sabías que es isósceles?

Carolina: Porque los radios son iguales y nada más íbamos a hacer dos líneas para que quedaran iguales.

Profesora: ¿Y por qué entonces tu compañera los midió? ¿Tocaba medirlos?

Carolina: No, porque ya ahí sirve que los radios son iguales.

Profesora: No había necesidad de medirlos, porque ya se sabía. Y si 
se agrandaba o achicaba la circunferencia, ¿qué pasaba?

Carolina: $\quad$ Ya, ya... este... cambiaban las medidas y ya no era triángulo isósceles.

Profesora: ¿ ¿No? ¿Si agrandamos el círculo?

Carolina: ¡Ah sí! Pero ya no queda de la misma medida de los primeros radios que hicimos.

Profesora: Pero, ¿sigue siendo triángulo isósceles, o no?

Carolina: Sí.

Profesora: ¿ ¿Cuál es la diferencia con el otro triángulo isósceles?

Carolina: Nada más cambia la medida.

Carolina reconoce la cualidad esencial como una propiedad geométrica general (dos lados congruentes) que determina al triángulo isósceles. Ella afirma que desde la construcción robusta realizada, donde dos radios de una circunferencia son los lados del triángulo, entonces son congruentes. Desde el punto de vista de la equidad, en el análisis de la resolución de este problema, encontramos que los estudiantes tuvieron la oportunidad de emplear nuevas herramientas de exploración (como la medida), modificar sus estrategias de intervención gracias a la retroalimentación proporcionada por el programa de geometría dinámica y la ayuda de la profesora, explicar sus producciones, producir enunciados generales y justificarlos. Asimismo, el equivocarse se vio como parte del proceso de resolución del problema planteado y una oportunidad para el aprendizaje de propiedades geométricas.

\section{Discusión}

Afirmamos que la experiencia narrada es un intento de trabajar en pro de la equidad. A lo largo del informe expuesto es posible evidenciar, además de lo ya señalado, que los estudiantes tuvieron oportunidades de trabajar de manera autónoma con el programa Cabri, explorando a su propio ritmo y con libertad para proponer construcciones y rehacerlas en caso de necesidad; apoyarse mutuamente en el manejo de programa y trabajar en equipo; expresar sus ideas, fruto de la exploración, explicarlas y justificarlas; e interactuar con la profesora en busca de apoyo para 
avanzar en su indagación. Sin duda alguna, el reconocimiento de la equidistancia entre puntos o de la congruencia de lados como propiedades de configuraciones geométricas, que permanecen invariantes a pesar de que otras propiedades se modifiquen, es un acercamiento más productivo, en términos matemáticos, que el estudio de las figuras en dibujos realizados a mano alzada, e incluso que construcciones hechas con regla y compás.

A partir de nuestro análisis, podemos asegurar que los estudiantes tuvieron la oportunidad de vivir una experiencia de actividad matemática productiva, en la que pudieron experimentar personalmente el ejercicio de suponer una vía para resolver un problema, ponerla a prueba, identificar dimensiones de variación, verificar propiedades y construir justificaciones para asegurar que su propuesta era válida. El interés con el que los estudiantes enfrentaron la resolución de los problemas, el hecho de que surgieran varias formas para resolverlos y las expresiones de sorpresa o agrado por obtener aquello que se les solicitaba son muestra del involucramiento en la actividad matemática. Asimismo, el esfuerzo realizado por algunos niños para construir justificaciones empleando hechos geométricos, más que argumentos empíricos, muestra que la trayectoria sugerida en el experimento es apropiada, aunque hacen falta muchos más esfuerzos en esa dirección y un trabajo sostenido durante mucho más tiempo.

Pese a lo dicho, a pesar de observar un avance de algunos estudiantes en los sucesivos ciclos de resolución de problemas, transitando entre las fases de inocencia, adquisición e internalización, son muy pocos los que dan muestras de encontrarse en algún momento en las dos últimas fases. Un sinnúmero de dificultades hace que el proceso de involucrar a estudiantes de primaria en el aprendizaje de las matemáticas, desde el punto de vista de construcción de significado de ideas abstractas, a partir del estudio de las dimensiones de variación de los objetos geométricos, sea una empresa compleja y de largo aliento. En particular, en el experimento de enseñanza observamos las dificultades que mencionamos a continuación, que requieren atención, en aras de tener un acceso equitativo al conocimiento.

Una dificultad tiene que ver con el hecho de que los estudiantes no han construido un criterio compartido sobre qué es una propiedad matemática y qué no. Esto hace que la atención que prestan a las cualidades de los objetos no se centre necesariamente en dimensiones de variación de tipo matemático. Al disponer de un ambiente de trabajo enriquecido con varios estímulos visuales, los estudiantes se enfocan en atributos como el color, la posición de objetos en la pantalla, los nombres de los puntos, etc., y no necesariamente en cualidades que el profesor espera que atiendan, como la equidistancia o la congruencia. Así, el diferenciar 
estas propiedades implica reconocer qué es lo importante para observar, desde el punto de vista matemático, lo cual requiere construir un criterio compartido al respecto.

Ligada con la anterior dificultad, se encuentra otra que tiene que ver con el tipo de explicaciones que se espera que los estudiantes den, cuando se trabaja en matemáticas. Desde el punto de vista del profesor, es obvio que las razones con las cuales explica el comportamiento de los objetos tienen que ver con sus atributos geométricos: distancia, equidistancia, congruencia, igual medida, por ejemplo. Sin embargo, los estudiantes pueden esgrimir otros argumentos, que no provienen necesariamente del mundo matemático. En nuestro experimento, fueron notorias explicaciones basadas en la percepción visual, tales como que dos lados son congruentes "porque se ven iguales" o dos puntos están a la misma distancia de otro "porque así se ven" o "cuando el punto se va a la derecha, la longitud es mayor". Pero también surgieron otras, que Ilamaron nuestra atención, porque provienen de una suerte de "realismo mágico" que rebasa las formas racionales de explicación, tales como: "estos segmentos se hacen uno solo" o "el punto se desaparece". No todos los niños aprendieron a discriminar cuáles de esas explicaciones son aceptables desde el punto de vista matemático. Esto hace que la construcción de criterios compartidos sobre lo que es una explicación matemática aceptable sea un reto para los profesores.

Una tercera dificultad tiene que ver con aquello que los estudiantes creen que se espera de ellos en la clase de matemáticas. Pese a insistirles que debían proponer sus propias vías para resolver los problemas, más de la mitad de ellos asumieron una actitud pasiva, esperando que la profesora les dijera "lo que tenían que hacer". Al preguntarles qué se les ocurría que podrían hacer, manifestaban que no lo sabían y no tomaban la iniciativa proponiendo cualquier idea, a pesar de la insistencia para que lo hicieran. Finalmente, solo si la profesora les insinuaba una vía, o miraban lo que hacían sus compañeros, procedían a hacer alguna construcción. Este hecho Ilama nuestra atención, pues solo si los estudiantes sienten que pueden proponer sus ideas y las expresan, se puede construir a partir de lo que saben o piensan.

También llamó nuestra atención el hecho de que la mayoría de los estudiantes no usaran el conocimiento "aprendido" al resolver un problema, en el siguiente. Por ejemplo, después de discutir ampliamente que si construían un triángulo isósceles haciendo una figura blanda, la congruencia de dos lados no se mantendría, y por lo tanto esa no era una buena vía para resolver el problema, prácticamente todos los grupos de estudiantes comenzaron a resolver el problema de construir un triángulo equilátero mediante una construcción blanda. ¿Qué hace que procedan así? ¿Es porque no tienen otro recurso de resolución del problema y quieren 
hacer la tarea, en todo caso? ¿Es porque no están acostumbrados a revisar qué de lo aprendido les puede servir? Este hecho hizo que el tránsito entre fases no se diera tan rápidamente como se presume en la teoría de la variación.

También es importante señalar las dificultades comunicativas de los estudiantes para expresar lo que estaban haciendo, qué habían encontrado, y cómo explicaban sus resultados. El asunto no es de timidez o de falta de lenguaje común entre los estudiantes y la profesora. Tiene que ver con la falta de prácticas escolares que fomenten en los niños no solo la expresión oral, sino también escrita.

\section{Conclusiones}

Tal como lo señalamos en la introducción, trabajar en pro de la equidad tiene que ver con buscar transformaciones en las prácticas escolares para favorecer el acceso a prácticas matemáticas poderosas. En consonancia con nuestro marco de referencia, una vía que posibilita dicho acceso es mediante la experimentación personal de situaciones en las que propiedades matemáticas se vivencien como dimensiones de variación. Esto hace que reivindiquemos el uso de programas de geometría dinámica en el aprendizaje de la geometría a temprana edad. Sin embargo, no basta introducir su uso en las clases. Este debe ir acompañado de discusiones sobre aquello que se hace, explicaciones, verificaciones y justificaciones, que permitan que los niños construyan criterios compartidos sobre el quehacer matemático.

El experimento de enseñanza confirma nuestra hipótesis sobre las bondades de generar ambientes de resolución de problemas, a partir de problemas abiertos de construcción, en los que los estudiantes puedan proponer vías de construcción y ganen conocimientos que puedan ser la base de información compartida para justificar nuevas construcciones. En particular, las propiedades de la circunferencia son una herramienta supremamente útil para disponer de un mecanismo de construcción, verificación y justificación, que está al nivel de estudiantes de primaria, y debería explotarse. La simple asociación entre una representación visual, acompañada de una definición, no es suficiente para construir un conocimiento útil posteriormente. Los niños deben construir circunferencias, usar circunferencias para construir otros objetos, estudiar propiedades de puntos de la circunferencia, estudiar propiedades de objetos inscritos en circunferencia, etc., como herramientas útiles para resolver problemas geométricos.

Existe una creencia generalizada acerca del impacto positivo del uso de tecnología digital con fines educativos. Se evidencia un reconocimiento social y político del importante efecto que pueden tener, por las características

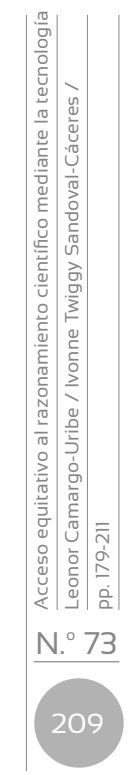


específicas de visualización, interactividad, simulación y dinamicidad para impulsar experiencias significativas de acceso al conocimiento. En diferentes ámbitos se plantea incluso que la tecnología digital ha democratizado el acceso al conocimiento matemático de niños y jóvenes y ha contribuido a elevar el nivel de desarrollo científico y tecnológico de nuestras sociedades. Sin embargo, a pesar de los esfuerzos por popularizar el acceso escolar a la tecnología digital, no parece que su uso haya contribuido aún a disminuir las desigualdades sociales y hay un cuestionamiento explícito a si estas tecnologías han contribuido a elevar o a disminuir la inequidad. Diversos marcos teóricos y herramientas metodológicas han surgido de la investigación, para orientar los diseños curriculares y alimentar la discusión sobre tal impacto. Esperamos que experiencias como la que comunicamos en este artículo contribuyan a la reflexión sobre las implicaciones reales del papel de la tecnología digital en las prácticas de enseñanza y aprendizaje de las matemáticas y fortalezcan el trabajo conjunto de educadores e investigadores en busca del impacto deseado.

\section{Agradecimientos}

Agradecimiento a la Secretaría de Relaciones Internacionales por la Beca de Excelencia de Programas Especiales del Gobierno de México para Extranjeros Convocatoria 2015, Amexid.

\section{Referencias}

Dunbar, K. y Klahr, D. (2013). Scientific thinking and reasoning. En K. Holyoak y R. Morrison (eds.). The Oxford handbook of thinking and reasoning (pp. 701-718). Nueva York: Oxford University Press.

Forgasz, H.; Vale, C. y Ursini, S. (2010). Technology for mathematics education: Equity, access and agency. En C. Hoyles, y J. B. Lagrange (eds.). (2010). Mathematics Education and technology - Rethinking the terrain. The $17^{\text {th }}$ ICMI Study. Nueva York: Springer.

Healy, L. (2000). Identifying and explaining geometrical relationship: Interactions with robust and soft Cabri constructions. En T. Nakahara y M. Koyama (eds.). Proceedings of the 24th Conference of the International Group for the Psychology of Mathematics Education (vol. 1, pp. 103-117). Hiroshima, Japón: Universidad de Hiroshima.

Hogan, K.; Nastasi, B. y Pressley, M. (2000). Discourse patterns and collaborative scientific reasoning in peer and teacher-guided discussions. Cognition and Instruction, 17(4), 379-432.

Jones, K. (2000). Providing a foundation for deductive reasoning: students' interpretation when using dynamic geometry software and their evolving mathematical explanations. Educational Studies in Mathematics, 44, 55-85. 
Laborde, C. (2000). Robust and soft constructions: Two sides of the use of dynamic geometry environments. University Joseph Fourier and Teacher Education Institute Grenoble.

Lawson, A. (2004). The nature and development of scientific reasoning: A synthetic view. International Journal of Science and Mathematics Education, 2(3), 307-338.

Leung, A. (2003). Dynamic geometry and the theory of variation. En N. Pateman, B. Dougherty, and J. Zilliox. (eds.) Proceedings of the $27^{\text {th }}$ Conference of the International Group for the Psychology of Mathematics Education (vol. 3, pp. 197-204). Honolulu, USA.

Leung, A. (2008). Dragging in a dynamic geometry environment through the lens of variation. International Journal of Computers for Mathematical Learning, 13, 135-157.

Leung, A. y López-Real, F. (2000). An analysis of students' explorations and constructions using Cabri geometry. En M. A. Clements, H. Tairab and K. Y. Wong (eds.), Science, Mathematics and Technical Education in the 20th and 21 st centuries (pp. 144-154). University Brunei Darussalam.

Mariotti, M.A. (2000). Introduction to proof: The mediation of a dynamic software environment. Educational Studies in Mathematics, 44, 25 53.

Marton, F.; Runesson, U.; Tsui, A. B. M. (2004). The space of learning. En F. Marton; A.B.M. Tsui (eds.) (2004). Classroom discourse and the space of learning. Nueva Jersey: Lawrence Erlbaum Associates.

Molina, M.; Castro, E.; Molina, J. L. y Castro, E. (2011). Un acercamiento a la investigación de diseño a través de los experimentos de enseñanza. Enseñanza de las Ciencias, 29(1), 75-88.

Olivero, F. (2003). The proving process within a dynamic geometry environment (PhD thesis). University of Bristol.

Perry, P.; Samper, C.; Molina, O.; Camargo, L.; Echeverry, A. (2012). La geometría del ángulo desde otro ángulo: una aproximación metodológica alternativa. Épsilon. Revista de Educación Matemática, 29(3), 82, 41-56.

Secretaría de Educación Pública. (2011). Programas de estudio 2011. Guía para el maestro. Educación Básica. Primaria. Quinto grado. México: Secretaría de Educación Básica.

Secretaría de Educación Pública. (2013). Desafíos. Quinto grado. Docente. México: Secretaría de Educación Básica.

Skovsmose, O. (1985). Mathematics education versus critical education. Educational Studies in Mathematics, 16(4), 337-354. 